\title{
A New Luminosity Effect in QSO Spectra
}

\author{
Michael R. Corbin
}

Steward Observatory, University of Arizona, Tucson, AZ 85721, USA

Todd A. Boroson

U.S. Gemini Program, National Optical Astronomy Observatories, P.O. Box 26732, Tucson, AZ 85726, USA

\begin{abstract}
Comparison of the emission-line and continuum parameters of 48 low-redshift QSOs reveals the asymmetry of the CIV $\lambda 1549$ broad emission line to be strongly correlated with ultraviolet continuum luminosity, such that increasing luminosity produces increasing redward asymmetry. A similar correlation has been found for broad $\mathrm{H} \beta$. Redward profile asymmetries can be modeled as the result of the gravitational redshift of line photons from the very broad-line region (VBLR) by $10^{9}-10^{10} M_{\odot}$ black holes, but blueward profile asymmetries require a competing effect such as electron scattering.
\end{abstract}

\section{Introduction}

Using data from the $H S T$ and $I U E$ archives, we have formed combined ultraviolet and optical spectra of 48 objects in the redshift range $0.034-0.774$. The sample contains 22 radio-quiet objects and 26 radio-loud objects, and covers approximately 2.5 decades in ultraviolet continuum luminosity. We measure and compare an array of emission-line and continuum parameters including emissionline equivalent width, velocity width, and asymmetry, along with the luminosity of the ultraviolet continuum as measured at the position of C IV $\lambda 1549$.

\section{Results and Interpretation}

The strongest of the new correlations revealed in our comparisons is between ultraviolet continuum luminosity and the asymmetry of the C IV $\lambda 1549$ profile, such that as continuum luminosity increases the profiles become more redward asymmetric. This is shown in Fig. 1, where the asymmetry index is defined as $\left(\lambda_{c}(3 / 4)-\lambda_{c}(1 / 4)\right) / \mathrm{FWHM}$, where $\lambda_{c}(3 / 4)$ and $\lambda_{c}(1 / 4)$ are the wavelength centroids of the profile at $3 / 4$ and $1 / 4$ maximum intensity. The continuum luminosities are measured for $H_{0}=50 \mathrm{~km} \mathrm{~s}^{-1} \mathrm{Mpc}^{-1}$ and $q_{0}=0$. This correlation is the same as found between the asymmetry of the broad $\mathrm{H} \beta$ profile and $2 \mathrm{keV}$ X-ray luminosity in an overlapping sample (Corbin 1993), and appears to be followed by both radio-loud and radio-quiet objects. The CIV $\lambda 1549$ asymmetry is also correlated with the full width at zero intensity (FWZI) of the Ly $\alpha$ profile 


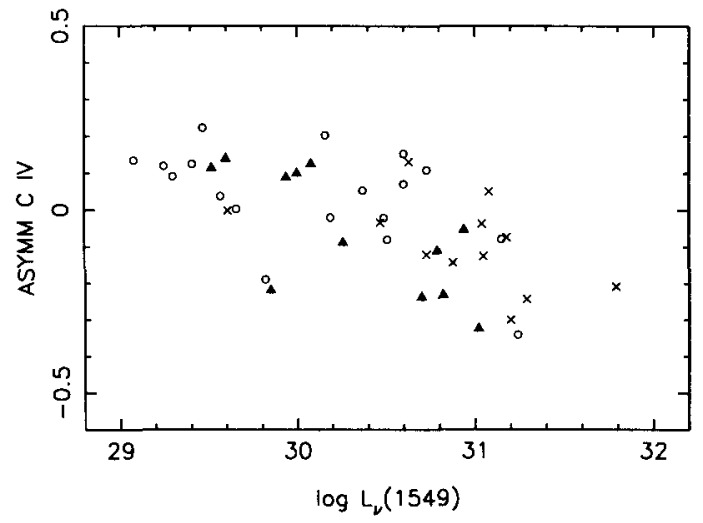

Figure 1. Correlation of ultraviolet continuum luminosity at $1549 \AA$ and CIV $\lambda 1549$ profile asymmetry. Open circles are radio-quiet objects, crosses are flat-radio spectrum quasars, triangles are steep-radio spectrum quasars.

in the sample objects, with broader profiles associated with stronger redward asymmetry. A similar relation holds for the asymmetry of $\mathrm{H} \beta$ and its FWHM (Boroson \& Green 1992).

Corbin (1995) and Popovic et al. (1995) find that redward profile asymmetries can be modeled as the gravitational redshift of emission from the very broad-line region (VBLR; see Brotherton et al. 1994) due to $\sim 10^{9-10} M_{\odot}$ black holes. If the VBLR gas is virialized, masses in the same range are inferred from the profile widths. The correlated increase in redward asymmetry, profile width, and continuum luminosity may thus represent a black-hole mass/luminosity relation of the type suggested by emission-line variability studies (Koratkar \& Gaskell 1991), provided that the size of the VBLR does not scale strongly with luminosity. Under this interpretation profile blueward asymmetries are the result of a competing effect acting to blueshift the line profiles, such as may be produced by electron scattering in a two-phase medium (Kallman \& Krolik 1986; Ferrara \& Pietrini 1993).

\section{References}

Boroson, T. A., \& Green, R.F. 1992, ApJS, 80, 109.

Brotherton, M.S., Wills, B.J., Francis, P. J., \& Steidel, C.C. 1994, ApJ, 430, 495.

Corbin, M. R. 1993 ApJ, 403, L9.

Corbin, M. R. 1995, ApJ, 447, 496.

Ferrara, A., \& Pietrini, P. 1993, ApJ, 405, 130.

Kallman, T.R., \& Krolik, J. H. 1986, ApJ, 308, 805.

Koratkar, A. P., \& Gaskell, C. M. 1991, ApJ, 370, L61.

Popovic, L. C., Vince, I., Atanackovic-Vukmanovic, O., \& Kubicela, A. 1995, A\&A, 293, 309 . 